\title{
Prevalence of type 2 diabetes, prediabetes, and gestational diabetes mellitus in women of childbearing age in Middle East and North Africa, 2000-2017: protocol for two systematic reviews and meta-analyses
}

\author{
Rami H. Al-Rifai (i) and Faisal Aziz
}

\begin{abstract}
Background: Diabetes mellitus (DM) in women of childbearing age may affect the fetus, thereby accelerating the intergenerational risk of DM. The Middle East and North Africa (MENA) region is experiencing a growing epidemic of DM. We aim to conduct two systematic reviews to summarize the burden of DM in women of childbearing age in the MENA region. In the systematic review 1, we aim to (1) systematically aggregate the evidence on type 2 DM (T2DM) and prediabetes and (2) to synthesize overall estimate on the prevalence of T2DM and overall estimate on the prevalence of prediabetes, in women of childbearing age (15-49 years). In the systematic review 2, we aim to (1) systematically aggregate the evidence on gestational DM (GDM) and (2) to synthesize overall estimate on the prevalence of GDM in pregnant women.
\end{abstract}

Methods: For systematic reviews 1 and 2, we will conduct a comprehensive search of the literature published in six electronic databases (MEDLINE-PubMed, EMBASE, Web of Science, SCOPUS, Cochrane Library, and Academic Search Complete). Variant and broad search terms will be designed to identify epidemiologic studies on the prevalence of T2DM and prediabetes in women of childbearing age, and GDM in pregnant women, published between 2000 and 2017. The MENA region will be defined according to the World Bank Country and Lending Groups. Retrieved citations will be screened, and data from the eligible research reports against specific eligibility criteria will be extracted. The findings of each systematic review will be reported separately following the Preferred Reporting Items for Systematic Reviews and Meta-Analyses Guidelines (PRISMA).

Discussion: Published literature on the prevalence of different types of DM among different population groups has been recently increased in the MENA region. This is the first review to fill an evidence gap on the overall burden, in the form of prevalence, of T2DM, prediabetes, and GDM in women of childbearing age in the MENA region. The findings of the two reviews will help in understanding the regional burden of these three types of DM in specific population groups to identify priority areas for interventions.

Systematic review registration: The PROSPERO registration number for the systematic review 1 is "CRD42017069231" dated 12/06/2017 and for the systematic review 2 is "CRD42018100629" dated 18/06/2018.

Keywords: Systematic review, Meta-analysis, Type 2 diabetes mellitus, Gestational diabetes mellitus, Middle East and North Africa region, MENA region

\footnotetext{
* Correspondence: rrifai@uaeu.ac.ae

Institute of Public Health, College of Medicine and Health Sciences, United

Arab Emirates University, P.O. Box 17666, Al Ain, UAE
}

(c) The Author(s). 2018 Open Access This article is distributed under the terms of the Creative Commons Attribution 4.0 International License (http://creativecommons.org/licenses/by/4.0/), which permits unrestricted use, distribution, and reproduction in any medium, provided you give appropriate credit to the original author(s) and the source, provide a link to the Creative Commons license, and indicate if changes were made. The Creative Commons Public Domain Dedication waiver (http://creativecommons.org/publicdomain/zero/1.0/) applies to the data made available in this article, unless otherwise stated. 


\section{Background}

Globally, non-communicable diseases (NCDs) are expected to contribute to over three quarters of all deaths in 2030 [1]. Diabetes mellitus (DM) ranks seventh of the NCDs world's biggest killers [1]. Worldwide, the number of individuals with DM is projected to rise by $48 \%$, from 425 million in 2017 to 629 million by 2045 [2]. Pronounced rise in the DM prevalence is projected to be in low- and middle-income countries including the African region (by $156 \%$, from 16 million in 2017 to 41 million in 2045), the South-East Asian region (by 84\%, from 82 million in 2017 to 151 million in 2045), followed by the Middle East and North African (MENA) region (by $72 \%$, from 39 million in 2017 to 67 million in 2045) [2]. One of the global targets is to halt, by 2025 , the rise in the age-standardized adult prevalence of DM at its 2010 level [3].

Type 2 DM (T2DM) is the most common type of DM, accounting for around $90 \%$ of all DM cases [4-6]. As the incidence of T2DM continues to rise and increasingly affects individuals of all ages [2], women of childbearing age, defined by the World Health Organization (WHO) as women aged between 15 and 49 years [7], are also affected by the global rise in DM epidemic. Gestational DM (GDM) affects pregnant women usually during the second and third trimesters of pregnancy though it can occur at any time during pregnancy [2]. Children of pregnant mothers with GDM are at a high risk of developing T2DM in adulthood [8], high blood pressure, or macrosomia [2]. It has been estimated that $75-90 \%$ of cases of hyperglycemia during pregnancy are related to GDM [9].

The burgeoning epidemics of T2DM and GDM have been attributed to rising levels of different modifiable risk factors: overweight and obesity driven by changes in lifestyle, a poor diet, and physical inactivity; smoking; and increase in consumption of sugar-sweetened beverages [10-13]. Worldwide, in 2016, more than 1.9 billion adults (aged $\geq 18$ years) were overweight $\left(\mathrm{BMI}>25 \mathrm{~kg} / \mathrm{m}^{2}\right)$; of these, over 650 million were obese $\left(B M I>30 \mathrm{~kg} / \mathrm{m}^{2}\right)$ [14]. An increase in the BMI is associated with an increased risk of developing T2DM or GDM [13, 15]. The overall relative risk of developing T2DM in obese and overweight individuals compared to those with a normal weight was estimated to be 7.19 and 2.99, respectively [16].

\section{Rationale}

In the MENA region that comprises 21 countries according to the World Bank and Lending Groups [17], women show a higher prevalence of overweight and obesity than men [18]. In the MENA region in 2013, the combined age-standardized prevalence of overweight and obesity among women $\geq 20$ years was $65.5 \%$ (obese $33.9 \%$ ) and among men $\geq 20$ years was $58.5 \%$ (obese 20.3\%) [18]. This high prevalence of overweight and obesity among women may affect and exacerbate the burden of T2DM and GDM in women, eventually increasing the risk of T2DM- and GDM-associated complications and pregnancy outcomes $[8,19]$.

Valid and consistent estimates of T2DM, prediabetes, and GDM prevalence among specific population groups in the MENA region are needed to develop effective interventions. Estimating the burden of T2DM and prediabetes in women of childbearing age, and GDM in pregnant women at regional level in adherence to the Guidelines for Accurate and Transparent Health Estimates Reporting (GATHER) [20], requires systematic reviews and meta-analyses of studies on the regional prevalence of T2DM and prediabetes in women of childbearing age and GDM in pregnant women.

Evidence on the rising burden of T2DM and prediabetes epidemics in women of childbearing age and GDM in pregnant women in the MENA region based on individual studies will be compiled and summarized in two systematic reviews and meta-analyses studies. The findings of the two reviews, for the first time, will fill an evidence gap to inform policy makers on the epidemiologic burden of T2DM, prediabetes, and GDM in childbearing age women to design tailored interventions. Here, we present the protocol for two systematic reviews to keep a clear scope for each of the two systematic reviews. In fact, as the methods of systematic reviews and meta-analyses studies are similar for the most part, this protocol for two systematic reviews would avoid publishing such similar and established methods in one more additional protocol. In this protocol, we refer separately to systematic reviews 1 and 2 . Each of these two reviews addresses different objectives, but they will be conducted in parallel and coherently.

\section{Aim}

The overarching aim of the systematic review 1 is to assess the regional burden, in the form of the prevalence, of T2DM and prediabetes in women of childbearing age 15-49 years while assessing the regional burden of GDM in pregnant women will be the aim of the systematic review 2, in the MENA region during the period of January, 2000, to June, 2017. The WHO criteria to diagnose different types of DM were updated over time in 1999 [21] and then in 2006 [22]. Although we are not limiting including studies based on the DM diagnosis criteria, the lower cutoff of the year 2000 will be used as studies conducted earlier may have used outdated criteria to ascertain the diagnosis of DM. To our knowledge, this is the first systematic review protocol for estimating the regional burden of T2DM, prediabetes, and GDM in women in childbearing age and pregnant women, in the MENA region. 


\section{Objectives}

\section{Systematic review 1}

The objectives of systematic review 1 are as follows:

1. To systematically review quantitative studies reporting the prevalence of T2DM or prediabetes in women of childbearing age in the MENA region, from January, 2000, to June, 2017.

2. To systematically synthesize the overall estimate on the prevalence of T2DM and the overall estimate on the prevalence of prediabetes in women of childbearing age in the MENA region, as reported in studies during the period January, 2000, to June, 2017.

\section{Systematic review 2}

The objectives of systematic review 2 are as follows:

1. To systematically review quantitative studies reporting the prevalence of GDM in pregnant women in the MENA region, as reported in studies during the period of January, 2000 to June, 2017.

2. To systematically synthesize the overall estimate on the prevalence of GDM in pregnant women in the MENA region, as reported in studies during the period of January, 2000 to June, 2017.

\section{Research questions}

Informed by the documented increase in the global epidemic of DM, our research questions for the systematic review 1 are (1) "what is the overall prevalence of T2DM?" and (2) "what is the overall prevalence of prediabetes?", in women of childbearing age in the MENA region, as reported in studies published during the period of January, 2000 to June, 2017. Systematic review 2 will answer the research question "what is the overall prevalence of GDM in pregnant women?", in the MENA region, as reported in studies published during the period of January, 2000, to June, 2017.

\section{Methods/design}

\section{Description of the outcomes}

The weighted mean prevalence of the following three outcomes will be assessed from studies to be included in the two systematic reviews.

\section{Systematic review 1}

The T2DM and prediabetes in women of childbearing age in the MENA region are the outcomes of interests.

\section{Systematic review 2}

In pregnant women, when the level of hyperglycemia first detected by testing at any time during the course of pregnancy meets the criteria for diagnosis of DM in the non-pregnant women, the condition is called DM in pregnancy [23]. DM in pregnancy is more likely to be detected as early as the first trimester. When hyperglycemia detected generally in the second trimester between 24 and 28 weeks of pregnancy does not meet the criteria of DM in pregnancy it is called GDM [23]. The GDM in pregnant women in the MENA region is the outcome of interest for this systematic review.

\section{Protocol and registration}

This protocol adheres to the Preferred Reporting items for Systematic Review and Meta-Analysis Protocols (PRISMA-P) statement (Additional file 1: Table S1) [24]. The two systematic reviews will be reported according to the PRISMA statement [25]. The protocol for systematic review 1 is registered online on PROSPERO "CRD42017069231" dated 12/06/2017 and for systematic review 2 "CRD42018100629" dated 18/06/2018, the international prospective register of systematic reviews.

\section{Eligibility criteria}

For both systematic reviews, we will include all epidemiologic studies reporting quantitative prevalence estimates according to the following inclusion and exclusion criteria:

Inclusion criteria

1. Study design: Observational epidemiologic studies including cross-sectional, population-based, comparative cross-sectional, and cohort studies.

2. Population: Women of childbearing age (15-49 years), regardless of the sample size and pregnancy status.

3. Geographical region: Any of the 21 countries of the MENA region, according to the definition of the World Bank Country and Lending Groups [17].

4. Language: Arabic or English.

5. DM ascertainment: Based on glucose level testing, medical records, or self-report supported with antiDM medications or a documented diagnosis.

6. Setting: No limitations. Hospital-based, populationbased, or clinic-based.

7. Publication period: January, 2000, to June, 2017. Exclusion criteria

1. Study design: Case-control, qualitative, and modeling studies; case reports and case series regardless of the number of cases; narrative reviews; conference abstracts with no full information or if authors have not responded to our inquiry on the full data; editorials; commentaries; letters to the editor; author replies; and other publications that do not include quantitative data on the prevalence of T2DM, prediabetes, or GDM. 
2. Population: Women age less than 15 years or older than 49 years. Studies in men and studies that combined men and women together.

3. Geographical region: Any country not located in the MENA region, according to the definition of the World Bank Country and Lending Groups [17].

4. Language: Non-Arabic or non-English.

5. DM ascertainment: Self-report not supported with anti-DM medications or a documented diagnosis.

6. Duplicate studies: Studies duplicating T2DM, prediabetes, or GDM ascertainment in the same population. In the case of duplicate publications, only the study containing the most information in the context of prevalence and ascertainment methodologies will be included.

7. Studies presenting contradictory/unclear quantitative measures that could not be verified with authors.

8. Studies including patients with T2DM, prediabetes, or GDM with other metabolic disorders or other NCDs in same category.

\section{Information sources}

For both systematic reviews, potentially eligible published articles (thereafter defined as a "research report") will be identified through searches in six electronic academic databases (MEDLINE, EMBASE, Web of Science, SCOPUS, Cochrane library, and Academic Search Complete; see Additional file 2: Box S1). The reference lists of retrieved eligible research reports for each of the two systematic reviews will be hand-searched for further studies that might have been missed.

\section{Search strategy}

A comprehensive and sensitive computerized search limited to the countries of the MENA region will be implemented to identify eligible published research reports for each of the two systematic reviews. We will use specific search terms based on Medical Subject Headings $(\mathrm{MeSH})$ and free-text (Text) search terms. The search terms will include variant terms on the DM combined using Boolean operators. The names of individual countries of the MENA region and regional variants will also be used to identify studies that may have been indexed under regional names. To ensure the comprehensiveness of the search strategy and not to miss any potential eligible studies, expert librarians in the National Medical Library at the United Arab Emirates University were consulted on the designed search strategy and on the searching process of the six targeted databases. A detailed search strategy with filters to be applied for both systematic reviews is presented in Additional file 2: Box S1.

\section{Screening strategy}

For both systematic reviews, the initial selection criteria will be broad to ensure that as many research reports as possible are assessed based on their relevance to each of the two systematic reviews. Any research report that are not relevant to any of the two systematic reviews will be excluded in the early stages of the title and abstract screening. The full-text research reports of seemingly relevant titles/abstracts will be accessed online. Research reports that are not freely available online will be obtained through the University Interlibrary Loan System. The full texts of research reports will be read for further screening based on the eligibility criteria. Two authors will independently screen the titles, abstracts, and full texts of potentially relevant research reports. Any conflicts between the two authors will be resolved by consensus or by consulting an expert.

\section{Removal of duplicates}

For both systematic reviews, the Endnote $\mathrm{X} 8$ reference manager database [26] will be used to manage the retrieval of articles and to screen for and exclude the duplicate reports. The following rules will be used to remove duplicate hits from the database:

1. Compare the title or various combinations of the author(s), year, secondary title, volume, issue, and page numbers with the "de-duplication" function.

2. Visually compare the full records of suspected duplicates.

3. Save duplicates in a separate database.

\section{First-level screening-title/abstract screening}

All titles/abstracts will be screened for relevance. Screened titles/abstracts will be classified into three categories: not relevant, relevant, or potentially relevant.

\section{Second-level screening-full-text screening}

The full texts of potentially relevant and completely relevant research reports identified during first-level screening will be retrieved and thoroughly evaluated to assess their eligibility. Irrelevant research reports against our eligibility criteria will be excluded.

\section{Data extraction}

For research reports found eligible to be included in systematic review 1 and systematic review 2, relevant data will be extracted into a predefined data extraction form, which will first be piloted using five research reports. The data will be extracted independently by two review investigators. Discrepancies between data extractors will be discussed and resolved to reach a consensus. If a consensus cannot be achieved, an expert will be consulted. 
The following parameters will be extracted from relevant studies: author names, date of publication, country where the study was conducted, number of participants, year of publication, study design, setting, target population, study period, methodology of outcome ascertainment, number of subjects enrolled, number of the tested women, age of the tested women, pregnant or child-bearing age, number of the women diagnosed with T2DM, prediabetes, or GDM, and prevalence of T2DM, prediabetes, or GDM. A complete list of the coded parameters to be extracted for both systematic reviews is shown in Additional file 2: Table S2.

\section{Data synthesis}

For both systematic reviews, research reports providing stratified estimates of the prevalence, the prevalence of the total sample will be replaced with pooling the stratified measures. A pre-defined sequential order will be followed when considering stratified prevalence measures. Prevalence estimates stratified according to BMI will be prioritized, followed by age, and year. This prioritization scheme will prioritize strata with more information on the tested subjects. Otherwise, the overall T2DM, prediabetes, or GDM prevalence measure will be included. In research reports stratifying prevalence of the outcome of interest at different levels (i.e., age and $\mathrm{BMI}$ ), one stratification level per included research report will be considered and included to avoid double-counting. If T2DM, prediabetes, or GDM was diagnosed using various ascertainment criteria, we will extract relevant information of the most sensitive and reliable ascertainment assay (i.e., prioritizing FBG over self-reported DM status).

\section{Risk of bias assessment}

We will evaluate the precision and the quality of each prevalence estimate measure identified in each of the two systematic reviews. We will assess each study's risk of bias (ROB) using the NIH tool for observational studies that has 14 criteria [27]. Also, we will use three additional quality criteria, namely, sampling methodology, ascertainment methodology, and response rate. These three additional criteria will assess the robustness of the implemented sampling methodology and the ascertainment methodology of the measured outcome(s). For each assessed criteria, each study has the potential to be categorized as "potentially of low ROB" if the answer was "yes" for that specific criteria, "potentially of high ROB" if the answer was "no" for that specific criteria, or "unclear" if the answer was neither "can't determine or not reported" for that specific criteria. A low ROB will be considered if the study/research report evaluated as a probability based, ascertained by biological assays, or with a response rate of $\geq 80 \%$.
To assess the precision of the reported prevalence estimates, a minimum sample size will be calculated using an established mathematical formula [28]. Each prevalence measure will be considered as having a high precision if the tested sample size was equal or higher than the calculated minimum sample size [28]. Items included to measure the $\mathrm{ROB}$ are listed in Additional file 2: Table S2. The ROB and precision will be reported for each research report not for each prevalence measure, unless different prevalence measures within the same research report were based on different methodologies such as a different sampling strategy.

\section{Summary measures and synthesis of results: meta-analysis}

For each systematic review, we will use the metaprop command to perform a meta-analysis of the prevalence estimates [29]. This command incorporates the Freeman-Tukey double arcsine transformation to stabilize the variances of prevalence measures [30, 31]. The prevalence measures will be weighted using the inverse variance method [31]. For each pooled estimate and its 95\% confidence interval (CI), a forest plot will be created to show the prevalence and corresponding 95\% CI for each study.

Several guidelines are available and implemented to ascertain different types of DM including the guidelines issued by the WHO in 1999 [21], WHO in 2006 [22], American Diabetes Association [32, 33], Carpenter and Coustan criteria [34], International Association of Diabetes and Pregnancy Study Groups [35], and National Diabetes Data Group Classification [36]. For both systematic reviews, the estimation of the pooled prevalence will be performed according to the ascertainment methodology and overall regardless of the ascertainment methodology. All analyses will be performed using the STATA SE15.0 statistical software [37].

For each systematic review, the heterogeneity in effect size will be evaluated across studies. Specifically, we will conduct Cochran's $Q$ test and extract several heterogeneity measures, including the estimate of the between-study variance of the true effect sizes using the $\tau$-squared statistic $\left(\tau^{2}\right)$, the magnitude and its $95 \%$ $\mathrm{CI}$ of the between-study variation that is due to heterogeneity rather than chance using the $I$-squared statistic $\left(I^{2}\right)$, and the $95 \%$ prediction interval which estimates the distribution of the true effect size among the included studies [38, 39]. The Q-statistic tests for heterogeneity are based on the null hypothesis that all studies share a common effect size. Hypothesis testing will be performed based on a $p$ value $<0.10$, implying that the studies do not share a common effect size [39]. 


\section{Assessment of meta-bias}

For each systematic review, we will assess the presence of meta-bias in terms of publication bias. To assess the publication bias, we will examine funnel plots supplemented with formal statistical testing using Egger's test [40]. The funnel plots of log (odds proportion) will be plotted against the sample size [41]. The asymmetry of the funnel plots will be examined by performing Egger's test [40] that is based on SE. To test the robustness of our findings, we will apply Duval and Tweedie's trim and fill methods [42].

\section{Additional analyses}

In each systematic review, if there is evidence for differences in prevalence estimates measures, the source of heterogeneity will be explored through subgroups analyses, according to DM-ascertainment criteria, using study-level characteristics such as sub-region, study period, year of publication, and precision. The MENA region will be divided into four sub-regions, including the North Africa region (Morocco, Algeria, Tunisia, Libya, Egypt, Malta, and Djibouti); Levant region (Jordan, Syria, Palestine, Israel, and Lebanon), Gulf Cooperation Council countries (United Arab Emirates, Qatar, Saudi Arabia, Kuwait, Yemen, Oman, and Bahrain), as well as Iran and Iraq. If feasible, sensitivity analyses will be conducted for studies with high precision or a low ROB. This may be complemented, where relevant, by meta-regression to further explain the heterogeneity. To construct a multivariable meta-regression, we will first evaluate the crude contribution of each factor to the log odds of the outcome prevalence at $p<0.10$. Then, we will sequentially add variables to the meta-regression starting with the variables showing the strongest correlation with the estimated prevalence separately, based on the univariate meta-regression analyses and relevance. The final regression model will include all variables that are significantly and independently associated with each of the measured three outcomes [38].

\section{Discussion}

Globally, the growing epidemic of diabetes and its increasing penetration in women of childbearing age have generated public health concerns on adverse pregnancy outcomes and accelerating the intergenerational risk of DM. The two systematic reviews to be conducted, to the best of our knowledge, will be the first to synthesis available data on T2DM and prediabetes in women of childbearing age and GDM in pregnant women, in the MENA region. Findings of the two reviews will fill an evidence gap in understanding the regional burden of T2DM, prediabetes, and GDM among specific population groups. The two systematic reviews will provide basis regional estimates for the future epidemiologic studies. Additionally, the two reviews will inform policy makers prioritize areas for interventions to improve maternal and child health outcomes in the MENA region.

\section{Additional files}

Additional file 1: Table S1. PRISMA-P 2015 checklist. Prevalence of type 2 diabetes, prediabetes, and gestational diabetes mellitus in women of childbearing age in Middle East and North Africa, 2000-2017: protocol for two systematic reviews and meta-analyses. (DOCX $33 \mathrm{~kb}$ )

Additional file 2: Box S1. Databases and search terms. Table S2. Data extraction parameters. (DOCX $44 \mathrm{~kb}$ )

\section{Abbreviations}

BMI: Body mass index; Cl: Confidence interval; DM: Diabetes mellitus; FBG: Fasting blood glucose; GATHER: Guidelines for Accurate and

Transparent Health Estimates Reporting; GDM: Gestational diabetes mellitus;

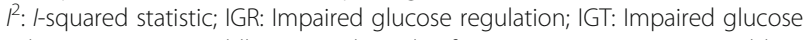
tolerance; MENA: Middle East and North Africa; NCDs: Non-communicable diseases; OGTT: Oral glucose tolerance test; Prediabetes: Prediabetes mellitus; PRISMA: Preferred reporting items for systematic reviews and meta-analyses guidelines; PRISMA-P: Preferred Reporting Items for Systematic Review and Meta-Analysis Protocols; ROB: Risk of bias; T2DM: Type 2 diabetes mellitus; UN: United Nations; WHO: World Health Organization; $\tau^{2}$ : $\tau$-squared statistic

\section{Acknowledgements}

We are grateful to the infrastructure provided by the Institute of Public Health, College of Medicine and Health Sciences at the United Arab Emirates University.

\section{Funding}

This protocol and the two systematic reviews to be conducted are funded by the Summer Undergraduate Research Experience (SURE) PLUS-Grant of the United Arab Emirates University, 2017 (Research grant: 31M348). The funder has no role in the development of this protocol.

\section{Authors' contributions}

RHA conceptualized and designed the study. FA contributed to protocol drafting and writing. RHA critically revised the protocol and approved the final version. RHA is the guarantor of the review. Both authors read and approved the final manuscript.

Ethics approval and consent to participate

Not applicable. This review will rely entirely on utilizing published data; thus, ethical approval will not be required.

Consent for publication

Not applicable.

\section{Competing interests}

The authors declare that they have no competing interests.

\section{Publisher's Note}

Springer Nature remains neutral with regard to jurisdictional claims in published maps and institutional affiliations.

Received: 15 January 2018 Accepted: 24 June 2018

Published online: 18 July 2018

\section{References}

1. World Health Organization. World Health Statistics 2008. http://www.who. int/whosis/whostat/EN_WHS08_Full.pdf?ua=1. Accessed 20 Nov 2017.

2. International Diabetese Fedration. International Diabetese Fedration Atals 2017 (8th edition). http://www.diabetesatlas.org/resources/2017-atlas.html. Accessed 20 Nov 2017. 
3. World Health Organization. Global action plan for the prevention and control of noncommunicable diseases 2013-2020. 2013. http://www.who. int/nmh/publications/ncd-action-plan/en/. Accessed 18 Dec 2017.

4. Evans JM, Newton RW, Ruta DA, MacDonald TM, Morris AD. Socio-economic status, obesity and prevalence of type 1 and type 2 diabetes mellitus. Diabet Med. 2000;17(6):478-80.

5. Holman N, Young B, Gadsby R. Current prevalence of type 1 and type 2 diabetes in adults and children in the UK. Diabet Med. 2015;32(9):1119-20.

6. Bruno G, Runzo C, Cavallo-Perin P, Merletti F, Rivetti M, Pinach S, et al. Incidence of type 1 and type 2 diabetes in adults aged 30-49 years: the population-based registry in the province of Turin, Italy. Diabetes Care. 2005; 28(11):2613-9.

7. World Health Organisation. Sexual and reproductive health. 2015. http:// www.who.int/reproductivehealth/topics/infertility/definitions/en/. Accessed 16 Nov 2017

8. Clausen TD, Mathiesen ER, Hansen T, Pedersen O, Jensen DM, Lauenborg J, et al. High prevalence of type 2 diabetes and pre-diabetes in adult offspring of women with gestational diabetes mellitus or type 1 diabetes: the role of intrauterine hyperglycemia. Diabetes Care. 2008;31(2):340-6. https://doi.org/ 10.2337/dc07-1596. PubMed PMID: 18000174.

9. Guariguata L, Linnenkamp U, Beagley J, Whiting DR, Cho NH. Global estimates of the prevalence of hyperglycaemia in pregnancy. Diabetes Res Clin Pract. 2014;103(2):176-85. https://doi.org/10.1016/j.diabres.2013.11.003.

10. Imamura F, O'Connor L, Ye Z, Mursu J, Hayashino Y, Bhupathiraju SN, et al. Consumption of sugar sweetened beverages, artificially sweetened beverages, and fruit juice and incidence of type 2 diabetes: systematic review, meta-analysis, and estimation of population attributable fraction. Br J Sports Med. 2016;50(8):496-504. https://doi.org/10.1136/bjsports-2016h3576rep. PubMed PMID: 27044603; PubMed Central PMCID: PMCPMC4853528.

11. Ashrafi M, Gosili R, Hosseini R, Arabipoor A, Ahmadi J, Chehrazi M. Risk of gestational diabetes mellitus in patients undergoing assisted reproductive techniques. Eur J Obstet Gynecol Reprod Biol. 2014;176:149-52. https://doi. org/10.1016/j.ejogrb.2014.02.009. PubMed PMID: 24630294

12. InterAct $C$, Romaguera $D$, Norat $T$, Wark PA, Vergnaud AC, Schulze MB, et al. Consumption of sweet beverages and type 2 diabetes incidence in European adults: results from EPIC-InterAct. Diabetologia. 2013;56(7):152030. https://doi.org/10.1007/s00125-013-2899-8. PubMed PMID: 23620057.

13. Bays HE, Chapman RH, Grandy S, Group SI. The relationship of body mass index to diabetes mellitus, hypertension and dyslipidaemia: comparison of data from two national surveys. Int J Clin Pract. 2007;61(5):737-47. https:// doi.org/10.1111/j.1742-1241.2007.01336.x. PubMed PMID: 17493087; PubMed Central PMCID: PMCPMC1890993.

14. Obesity and overweight. Media centre. World Health Organization. Updated October 2017. http://www.who.int/mediacentre/factsheets/fs311/en/. Accessed 7 Jan 2018.

15. Hu FB, Manson JE, Stampfer MJ, Colditz G, Liu S, Solomon CG, et al. Diet, lifestyle, and the risk of type 2 diabetes mellitus in women. N Engl J Med. 2001;345(11):790-7. https://doi.org/10.1056/NEJMoa010492. PubMed PMID: 11556298

16. Abdullah A, Peeters A, de Courten M, Stoelwinder J. The magnitude of association between overweight and obesity and the risk of diabetes: a meta-analysis of prospective cohort studies. Diabetes Res Clin Pract. 2010; 89(3):309-19. https://doi.org/10.1016/j.diabres.2010.04.012. PubMed PMID: 20493574.

17. World Bank Country and Lending Groups. https://datahelpdesk.worldbank. org/knowledgebase/articles/906519-world-bank-country-and-lendinggroups. Accessed 10 Nov 2017.

18. Ng M, Fleming T, Robinson M, Thomson B, Graetz N, Margono C, et al. Global, regional, and national prevalence of overweight and obesity in children and adults during 1980-2013: a systematic analysis for the Global Burden of Disease Study 2013. Lancet. 2014;384(9945):766-81. https://doi. org/10.1016/S0140-6736(14)60460-8. PubMed PMID: 24880830; PubMed Central PMCID: PMCPMC4624264.

19. Elkholi DGEY, Nagy HM. The effects of adipocytokines on the endocrinometabolic features and obstetric outcome in pregnant obese women with polycystic ovary syndrome. Middle East Fertility Society J. 2014;19:292-302.

20. Stevens GA, Alkema L, Black RE, Boerma JT, Collins GS, Ezzati M, et al. Guidelines for accurate and transparent health estimates reporting: the GATHER statement. Epidemiol Serv Saude. 2017;26(1):215-22. https://doi. org/10.5123/S1679-49742017000100023. PubMed PMID: 28226024.
21. World Health Organization. Dept. of Noncommunicable Disease Surveillance. Definition, diagnosis and classification of diabetes mellitus and its complications. Geneva: World Health Organization; 1999.

22. Definition and diagnosis of diabetes mellitus and intermediate hyperglycemia: report of a WHO/IDF consultation. 2006. http://www.who.int/diabetes/ publications/Definition\%20and\%20diagnosis\%20of\%20diabetes_new.pdf. Accessed 13 Oct 2017

23. Hod M, Kapur A, Sacks DA, Hadar E, Agarwal M, Di Renzo GC, et al. The International Federation of Gynecology and Obstetrics (FIGO) initiative on gestational diabetes mellitus: a pragmatic guide for diagnosis, management, and care, Int J Gynaecol Obstet. 2015:131(Suppl 3):S173-S211. https://doi. org/10.1016/S0020-7292(15)30007-2. PubMed PMID: 26433807

24. Preferred reporting items for systematic review and meta-analysis protocols (PRISMA-P) 2015: elaboration and explanation. BMJ. 2016;354:i4086. https:// doi.org/10.1136/bmj.i4086. PubMed PMID: 27444514

25. Liberati A, Altman DG, Tetzlaff J, Mulrow C, Gotzsche PC, loannidis JP, et al. The PRISMA statement for reporting systematic reviews and meta-analyses of studies that evaluate health care interventions: explanation and elaboration. PLoS Med. 2009;6(7):e1000100. https://doi.org/10.1371/journal. pmed.1000100. PubMed PMID: 19621070; PubMed Central PMCID: PMCPMC2707010.

26. Reuter Thomson. EndNote X7 2017.

27. National Heart, Lung, and Blood Institute. Quality assessment tool for observational cohort and cross-sectional studies. https://www.nhlbi.nih.gov/ health-topics/study-quality-assessment-tools. Accessed 10 May 2018.

28. Clopper CJ, Pearson ESP. The use of confidence or fiducial limits illustrated in the case of the binomial. Biometrika. 1934;26:413. Epub

29. Nyaga VN, Arbyn M, Aerts M. Metaprop: a Stata command to perform metaanalysis of binomial data. Arch Public Health. 2014;72(1):39. https://doi.org/ 10.1186/2049-3258-72-39. PubMed PMID: 25810908; PubMed Central PMCID: PMCPMC4373114

30. Freeman MF, Tukey JW. Transformations related to the angular and the square root. Ann Math Stat. 1950;21(4):607-11.

31. Miller JJ. The Inverse of the Freeman - Tukey Double Arcsine Transformation. Am Stat. 1978;32(4):138. https://doi.org/10.1080/00031305.1978.10479283.

32. American Diabetes Association. (2) Classification and diagnosis of diabetes. Diabetes Care. 2015;38(Suppl):S8-S16.

33. American Diabetes Association. Diagnosis and classification of diabetes mellitus. Diabetes Care. 2006;29(Suppl 1):S43-8.

34. Carpenter MW, Coustan DR. Criteria for screening tests for gestational diabetes. Am J Obstet Gynecol. 1982;144(7):768-73. PubMed PMID: 7148898.

35. International Association of Diabetes, Pregnancy Study Groups Consensus P, Metzger BE, Gabbe SG, Persson B, Buchanan TA, et al. International association of diabetes and pregnancy study groups recommendations on the diagnosis and classification of hyperglycemia in pregnancy. Diabetes Care. 2010;33(3):676-82. https://doi.org/10.2337/dc09-1848. PubMed PMID: 20190296; PubMed Central PMCID: PMCPMC2827530.

36. National Diabetes Data Group. Classification and diagnosis of diabetes mellitus and other categories of glucose intolerance. Diabetes. 1979;28(12): 1039-57. PubMed PMID: 510803.

37. StataCorp. Stata statistical software: release 15. College Station: StataCorp LLC; 2017.

38. Borenstein MHL, Higgins JP, Rothstein HR. Introduction to meta-analysis. Chichester: Wiley; 2009.

39. Higgins JP, Thompson SG, Spiegelhalter DJ. A re-evaluation of randomeffects meta-analysis. J R Stat Soc Ser A Stat Soc. 2009;172(1):137-59. https://doi.org/10.1111/j.1467-985X.2008.00552.x. PubMed PMID: 19381330; PubMed Central PMCID: PMCPMC2667312.

40. Egger M, Smith GD. Meta-analysis. Potentials and promise. BMJ. 1997; 315(7119):1371-4. PubMed PMID: 9432250; PubMed Central PMCID: PMCPMC2127866.

41. Hunter JP, Saratzis A, Sutton AJ, Boucher RH, Sayers RD, Bown MJ. In metaanalyses of proportion studies, funnel plots were found to be an inaccurate method of assessing publication bias. J Clin Epidemiol. 2014;67(8):897-903. https://doi.org/10.1016/j.jclinepi.2014.03.003. PubMed PMID: 24794697.

42. Duval S, Tweedie R. Trim and fill: a simple funnel-plot-based method of testing and adjusting for publication bias in meta-analysis. Biometrics. 2000; 56(2):455-63. PubMed PMID: 10877304 